\title{
ON THE HAHN-MAZURKIEWICZ THEOREM IN NONMETRIC SPACES
}

\author{
SIBE MARDEŠIĆ
}

About $1914 \mathrm{H}$. Hahn [2] and S. Mazurkiewicz [4 $]^{1}$ topologically characterized continuous images of the real line segment as metric compact connected and locally connected spaces. This included the result that, for locally connected metric compacta, connectedness and pathwise connectedness coincide. ${ }^{2}$ These theorems were never extended to nonmetric spaces, although there existed a conjecture seemingly known to many topologists. ${ }^{3}$ The real line segment is replaced by ordered continua, while the pathwise connectedness is replaced by connectedness by ordered continua (for definitions see $\S 1)$. The conjecture can be stated as follows:

For Hausdorff compact and locally connected spaces, connectedness and connectedness by ordered continua coincide. The class of Hausdorff spaces, which are continuous images of ordered continua, and the class of Hausdorff compact connected and locally connected spaces coincide.

This paper refutes both parts of the conjecture by producing a counter-example, which is effectively constructed.

1. Statement of results. An ordered space is a topological space provided with a total ordering < such that the topology of the space is the order topology ${ }^{4}$ induced by $<$. An ordered continuum $C$ is an ordered space which is compact and connected. Each closed subset $C^{\prime} \subset C$ has a maximal and a minimal element, which are denoted by $\max C^{\prime}$ and $\min C^{\prime}$ respectively. In particular, we have the two endpoints $c_{0}=\min C$ and $c_{1}=\max C ; c_{0}<c_{1}$ unless $C$ is degenerate. The set $\left\{t \mid t \in C, t_{0} \leqq t \leqq t_{1}\right\}$ will be denoted by $\left[t_{0}, t_{1}\right]_{c}$.

Definition. A space $X$ is said to be connected by ordered continua provided, for each pair of points $x_{0} \in X, x_{1} \in X$, there is an ordered continuum $C$ and a map $\chi: C \rightarrow X$, mapping the end-points of $C$ into $x_{0}$ and $x_{1}$ respectively.

Presented to the Society, January 29, 1960; received by the editors October 19, 1959.

${ }^{1}$ On p. 166 of [4] references are given to three earlier Polish notes by S. Mazurkiewicz on the same subject.

${ }^{2}$ For an exposition see e.g. [5, Chapter III].

3 The author is indebted to Professor R. D. Anderson for bringing the conjecture to author's attention. The conjecture seems to be nowhere in print. As a very natural question it has occurred independently at least to E. Dyer, A. J. Ward and R. F. Williams.

See e.g. [3, p. 57]. 
THEOREM 1. There exists a Hausdorff compact and locally connected space $S$, which is connected but is not connected by ordered continua.

TheOREM 2. There exists a Hausdorff compact connected and locally connected space $S$, which is not obtainable as the image of a mapping $\chi: C \rightarrow S, C$ being an ordered continuum. ${ }^{5}$

A space $X$, which admits a mapping $\chi$ of an ordered continuum $C$ onto $X$, clearly, is connected by ordered continua. Therefore, a space $S$ which verifies Theorem 1, verifies at the same time Theorem 2 , too. The rest of the paper is devoted to constructing a space $S$ that verifies Theorem 1 .

2. Scheme for the construction of $S$. 1. Let $\left\{S_{r}\right\}, r \in R$, be a collection of topological spaces and let for each $r \in R$ be given a map $\rho_{r}: S_{r} \rightarrow I$ onto the real line segment $I=\{u \mid 0 \leqq u \leqq 1\}$. With each such family $\left\{S_{r}, \rho_{r}\right\}, r \in R$, we associate a space $S=S\left(S_{r}, \rho_{r}\right)$, defined as the subset of the Cartesian product $\prod_{r} S_{r}, r \in R$, consisting precisely of those points $s=\left\{s_{r}\right\} \in \prod_{r} S_{r}$, for which

$$
\rho_{r}\left(s_{r}\right)=\rho_{r^{\prime}}\left(s_{r^{\prime}}\right),
$$

for all $r, r^{\prime} \in R$. In other words, if $\pi_{r}:\left(\prod_{r} S_{r}\right) \rightarrow S_{r}$ denotes the projection onto $S_{r}$, then $S\left(S_{r}, \rho_{r}\right)$ is the maximal subset of $\prod_{r} S_{r}$ for which $\rho_{r} \pi_{r}:\left(\prod_{r} S_{r}\right) \rightarrow I$ is independent of $r \in R$. Henceforth we denote $\rho_{r} \pi_{r} \mid S$ by $\zeta . S$ is, clearly, a closed subset of $\prod_{r} S_{r}$; hence, if all $S_{r}$ are Hausdorff compact spaces, then so is $S$.

2. In the sequel we shall define a certain family $\left\{S_{r}, \rho_{r}\right\}, r \in R$, with the following property:

Property (i). Given any ordered continuum $C$ and a map $\phi: C \rightarrow I$, carrying the two end-points of $C$ into 0 and 1 respectively, then one can find an $r^{\prime} \in R$ (depending on $\phi$ ) such that there is no continuous $\operatorname{map} \psi_{r^{\prime}}: C \rightarrow S_{r^{\prime}}$ with $\rho_{r^{\prime}} \psi_{r^{\prime}}=\phi$.

Lemma 1. The space $S=S\left(S_{r}, \rho_{r}\right)$ associated with a family $\left\{S_{r}, \rho_{r}\right\}$, $r \in R$, which possesses Property (i) cannot be connected by ordered continua.

Proof. $\zeta: S \rightarrow I$ being a mapping onto, there exist points $s_{0} \in \zeta^{-1}(0)$ $C S$ and $s_{1} \in \zeta^{-1}(1) \subset S$. Suppose that $C$ is an ordered continuum and $\chi: C \rightarrow S$ a map with $\chi\left(c_{0}\right)=s_{0}, \chi\left(c_{1}\right)=s_{1}$. Then $\zeta \chi: C \rightarrow I$ is a map with

- Added in proof: Since the submitting of this paper very simple examples establishing Theorem 2 have been given in the author's paper, Mapping ordered continua onto product spaces, to appear in Glasnik Mat.-Fiz. Astr. Društvo Mat. Fiz. Hrvatske. Ser. II. vol. 15 (1960). Thus it appears that the hard question is the one settled by Theorem 1. 
$\zeta \chi\left(c_{0}\right)=0, \zeta \chi\left(c_{1}\right)=1$. By Property (i), there is an $r^{\prime} \in R$ such that there is no continuous mapping $\psi_{r^{\prime}}: C \rightarrow S_{r^{\prime}}$ with $\rho_{r^{\prime}} \psi_{r^{\prime}}=\zeta \chi$. However, this relation is verified by $\psi_{r^{\prime}}=\pi_{r^{\prime}} \chi$ because $\zeta=\rho_{r^{\prime}} \pi_{r^{\prime}}$. This contradiction proves the assertion.

In order to obtain Theorem 1 we have to impose further conditions on $\left\{S_{r}, \rho_{r}\right\}, r \in R$, which shall insure compactness, connectedness and local connectedness of $S$.

3. First, assume that all $S_{r}$ are Hausdorff compact spaces, while $R$ is infinite. Observe that $\prod_{r} S_{r}, r \in R$, is the inverse limit of all the finite Cartesian products $S_{r(1)} \times \cdots \times S_{r(n)},\{r(1), \cdots, r(n)\} \subset R$, the bonding maps of the inverse system being the obvious projections induced by inclusions $\{r(1), \cdots, r(n)\} \subset\left\{r^{\prime}(1), \cdots, r^{\prime}\left(n^{\prime}\right)\right\}$, $1 \leqq n<n^{\prime}$; these maps will be denoted by $\pi_{r(1)} \cdots r(n), r^{\prime}(1) \cdots r^{\prime}\left(n^{\prime}\right)$. It is readily seen that this inverse system presentation for $\prod_{r} S_{r}, r \in R$, induces a presentation for $S \subset \prod_{r} S_{r}$, where the spaces $S_{r(1)} \times \cdots$ $\times S_{r(n)}$ have to be replaced by the subsets $S_{r(1)} \ldots r(n)=S\left(S_{r(i)}, \rho_{r(i)}\right), i$ $=1, \cdots, n$, of $S_{r(1)} \times \cdots \times S_{r(n)}$; the bonding maps are restrictions of $\pi_{r(1)} \cdots r(n), r^{\prime}(1) \cdots r^{\prime}\left(n^{\prime}\right)$.

LemmA 2. If the maps $\rho_{r}: S_{r} \rightarrow I, r \in R$, are monotone, then the maps $\pi_{r(1) \cdots r(n), r^{\prime}(1) \cdots r^{\prime}\left(n^{\prime}\right)}: S_{r^{\prime}(1) \cdots r^{\prime}\left(n^{\prime}\right)} \rightarrow S_{r(1)} \cdots r(n)$ are monotone too.

Proof. Let $\{r(1), \cdots, r(n)\} \subset\left\{r^{\prime}(1), \cdots, r^{\prime}\left(n^{\prime}\right)\right\}, 1 \leqq n<n^{\prime}$; one can assume that $r^{\prime}(i)=r(i)$, for $i=1, \cdots, n<n^{\prime}$. If $s_{r(1)} \times \cdots \times s_{r(n)}$ $\in S_{r(1) \cdots r(n)}$, then clearly

$$
\begin{aligned}
& \left(\pi_{r(1)} \cdots r(n), r^{\prime}(1) \cdots r^{\prime}\left(n^{\prime}\right)\right)^{-1}\left(s_{r(1)} \times \cdots \times s_{r(n)}\right) \\
& \quad=s_{r(1)} \times \cdots \times s_{r(n)} \times\left(\rho_{r^{\prime}(n+1)}\right)^{-1}(u) \times \cdots \times\left(\rho_{r^{\prime}\left(n^{\prime}\right)}\right)^{-1}(u),
\end{aligned}
$$

where $u=\rho_{r(i)}\left(s_{r(i)}\right), i=1, \cdots, n$. Since all $\rho_{r^{\prime}}$ are monotone maps (by assumption), it follows that the set (2) is connected as required.

Now, we state the second property required for $\left\{S_{r}, \rho_{r}\right\}, r \in R$ :

Property (ii). All the maps $\rho_{r}, r \in R$, are monotone and all the spaces $S_{r(1)} \ldots r(n), 1 \leqq n$, are Hausdorff compact connected and locally connected.

In view of the preceding inverse system presentation for $S$ and Lemma 2, we see that whenever $\left\{S_{r}, \rho_{r}\right\}, r \in R$, has Property (ii), then $S=S\left(S_{r}, \rho_{r}\right)$ is the inverse limit of an inverse system of connected and locally connected Hausdorff compact spaces with bonding maps all of which are monotone mappings onto. A known theorem asserts that under these conditions the limit space is itself Hausdorff compact connected and locally connected (see [1, Theorem 4.3, p. 241]).

We summarize the results of this section in 
Lemma 3. Let $\left\{S_{r}, \rho_{r}\right\}, r \in R$, be an infinite family of spaces $S_{r}$ and maps $\rho_{r}: S_{r} \rightarrow I$ onto $I$, which possesses Properties (i) and (ii). Then the associated space $S=S\left(S_{r}, \rho_{r}\right)$ has all the properties required in Theorem 1.

It remains to show the existence of families $\left\{S_{r}, \rho_{r}\right\}, r \in R$, having Properties (i) and (ii).

3. The family $\left\{S_{r}, \rho_{r}\right\}, r \in R$. 1. The SeTs $K_{q}$ AND $M_{q}$. Consider the coordinate 3 -space with points $(u, v, w)$ and denote by $p_{u}, p_{v}$ and $p_{w}$ the projections on the $u, v$ and $w$ axes respectively. With each real number $q, 0<2 q<1$, we associate the broken line $K_{q}$, which consists of three straight line segments joining subsequently the points $a=(0,0,0), b=(1-q, 0,0), c=(q, 1,0), d=(1,1,0)$. We denote the corresponding segments by $a b, b c$ and $c d . K_{q}$ belongs to the unit square $I^{2} \times 0=\{(u, v, 0) \mid 0 \leqq u, v \leqq 1\}$.

Let $a^{\prime}, b^{\prime}, c^{\prime}, d^{\prime}$ denote the points $a \times 1, b \times 1$ etc. $\left(p_{u}\left(a^{\prime}\right)=p_{u}(a)\right.$, $p_{v}\left(a^{\prime}\right)=p_{v}(a)$ and $\left.p_{v}\left(a^{\prime}\right)=1\right)$. Consider the segments $a b, b^{\prime} c^{\prime}, b c$ and $c^{\prime} d^{\prime}$. Given a $u \in[q, 1-q]_{I}$, each of these four segments contains precisely one point, whose first coordinate equals $u$; denote these four points by $\alpha_{u}, \beta_{u}, \gamma_{u}$ and $\delta_{u}$ respectively. Finally, consider the straight line segments $\alpha_{u} \beta_{u}$ and $\gamma_{u} \delta_{u}$ joining $\alpha_{u}$ with $\beta_{u}$ and $\gamma_{u}$ with $\delta_{u}$ respectively. Now, define a subset $M_{q}$ of the unit cube $I^{3}$ by setting

$$
M_{q}=\left(K_{q} \times I\right) \cup\left(\cup_{u}\left(\alpha_{u} \beta_{u} \cup \gamma_{u} \delta_{u}\right)\right), \quad u \in[q, 1-q]_{I} .
$$

It is readily seen that $M_{q}$ is a connected compact (curvilinear) polyhedron, hence, a continuous curve. Furthermore, $p_{u}$ restricted to $M_{q}$ is a monotone mapping onto $I\left(p_{u}^{-1}(u)\right.$ is a broken line consisting of 1,3 or 5 edges).

2. The SET $N_{(q(n))}$. Consider again the unit square $I^{2} \times 0$ and the sequence of points $a_{n}=\left(1-1 / 2^{n}, 1-1 / 2^{n}, 0\right), n=0,1, \cdots$, converging towards $a_{\infty}=(1,1,0)$. Let $Q_{n} \subset I^{2} \times 0$ be the square with sides parallel to those of $I^{2} \times 0$ and having the points $a_{n}, a_{n+1}$ for a pair of opposite vertices. Let $\lambda_{n}: I^{3} \rightarrow Q_{n} \times I$ be the linear mapping carrying the points $(0,0,0),(1,0,0),(0,1,0)$ and $(0,0,1)$ into $a_{n}$, $\left(p_{u}\left(a_{n+1}\right), p_{v}\left(a_{n}\right), 0\right),\left(p_{u}\left(a_{n}\right), p_{v}\left(a_{n+1}\right), 0\right)$ and $a_{n} \times 1$ respectively.

Given any sequence $(q(n)), n=0,1, \cdots$, of reals $0<2 q(n)<1$, we consider the sets $\lambda_{n}\left(M_{q(n)}\right) \subset Q_{n} \times I$ and put

$$
N_{(q(n))}=N_{q(0) \ldots q(n)} \ldots=\bigcup_{n=0}^{\infty} \lambda_{n}\left(M_{q(n)}\right) \cup\left(a_{\infty} \times I\right) .
$$

It is readily seen that $N_{(q(n))}$ is a continuous curve and that $p_{u} \mid N_{(q(n))}$ is monotone (recall that $p_{u} \mid M_{q}$ is monotone). 
3. Definition of the family $\left\{S_{r}, \rho_{r}\right\}, r \in R$. Now, we define a family $\left\{S_{r}, \rho_{r}\right\}, r \in R$, which, we claim, possesses both Properties (i) and (ii). $R$ is the set of all sequences $(q(n)), n=0,1, \cdots$, of reals, $0<2 q(n)$ $<1$. If $r=(q(n)) \in R$, then $S_{r}=N_{(q(n))}$ and $\rho_{r}=p_{u} \mid N_{(q(n)) \text {. }}$

Lemma 4. The family $\left\{S_{r}, \rho_{r}\right\}, r \in R$, defined above possesses Property (i).

The proof will be carried through in several steps.

Lemma 5. Let $C$ be an ordered continuum with end-points $c_{0}<c_{1}$ and let $\phi: C \rightarrow I=\{u \mid 0 \leqq u \leqq 1\}$ be a map with $\phi^{-1}(0)=\left\{c_{0}\right\}, \phi^{-1}(1)=\left\{c_{1}\right\}$. Then there exists a real number $\eta(\phi), 0 \leqq \eta(\phi)<1$, such that $t_{1}, t_{2} \in C$, $t_{1} \leqq t_{2}$, implies $\phi\left(t_{1}\right)-\phi\left(t_{2}\right) \leqq \eta(\phi)$.

Proof. Consider the set $\Delta=\left\{\left(t_{1}, t_{2}\right) \mid t_{1}, t_{2} \in C, t_{1} \leqq t_{2}\right\}$; as a closed subset of $C \times C, \Delta$ is compact. Therefore, the mapping $\Phi: \Delta \rightarrow I$, defined by

$$
\Phi\left(t_{1}, t_{2}\right)=\phi\left(t_{1}\right)-\phi\left(t_{2}\right),
$$

has a maximum. Let

$$
\eta(\phi)=\max \Phi\left(t_{1}, t_{2}\right)=\Phi\left(t_{1}^{\prime}, t_{2}^{\prime}\right)
$$

for some $\left(t_{1}^{\prime}, t_{2}^{\prime}\right) \in \Delta$. Clearly, $0 \leqq \eta(\phi) \leqq 1$. However, $\eta(\phi)=\phi\left(t_{1}^{\prime}\right)$ $-\phi\left(t_{2}^{\prime}\right)=1$ would imply $\phi\left(t_{1}^{\prime}\right)=1, \phi\left(t_{2}^{\prime}\right)=0, t_{1}^{\prime} \leqq t_{2}^{\prime}$, contradicting the assumptions $\phi^{-1}(0)=\left\{c_{0}\right\}, \phi^{-1}(1)=\left\{c_{1}\right\}$.

Lemma 6. Let $\phi: C \rightarrow I$ be as in Lemma 5 and let $0<2 q<1-\eta(\phi)$. Then there is no continuous map $\psi: C \rightarrow K_{q}$ with $p_{u} \psi=\phi$.

Proof. Assume that $\psi$ is such a map. Then $p_{u} \psi\left(c_{0}\right)=\phi\left(c_{0}\right)=0$ and thus $\psi\left(c_{0}\right)=a$; similarly, $\psi\left(c_{1}\right)=d$. Since $C$ is connected and $K_{q}$ is an arc with end-points $a$ and $d, \psi$ is a map onto $K_{q}$ and thus $\psi^{-1}(b)$ is not empty. Let $t_{1}^{\prime}=\max \psi^{-1}(b)$. Then, clearly, $\psi$ maps $\left[t_{1}^{\prime}, c_{1}\right]_{c}$ into $b c \cup c d \subset K_{q}$, which implies the existence of $t_{2}^{\prime} \geqq t_{1}^{\prime}$ with $\psi\left(t_{2}^{\prime}\right)=c$. In other words, we have $\left(t_{1}^{\prime}, t_{2}^{\prime}\right) \in \Delta$ and $\Phi\left(t_{1}^{\prime}, t_{2}^{\prime}\right)=p_{u} \psi\left(t_{1}^{\prime}\right)-p_{u} \psi\left(t_{2}^{\prime}\right)$ $=p_{u}(b)-p_{u}(c)=1-2 q>\eta(\phi)$. This contradicts Lemma 5 .

Lemma 7. Let $\phi: C \rightarrow I$ be as in Lemma 5, let $0<2 q<1-\eta(\phi)$ and let $\psi: C \rightarrow M_{q}$ be a mapping with $p_{u} \psi=\phi$. Then there exist two points $t_{1}, t_{2} \in C$ such that $p_{w} \psi\left(t_{1}\right)=0, p_{w} \psi\left(t_{2}\right)=1$.

Proof. Assume that $p_{w} \psi(t) \neq 0$, for all $t \in C$. Define a mapping $\theta:\left(M_{q}-K_{q}\right) \rightarrow K_{q} \times 1$ as follows. For $(u, v, w) \in K_{q} \times(0,1]_{I}$, let $\theta(u, v, w)=(u, v, 1)$; for $(u, v, w) \in \alpha_{u} \beta_{u}$, let $\theta(u, v, w)=\beta_{u}$ and for $(u, v, w) \in \gamma_{u} \delta_{u}$, let $\theta(u, v, w)=\delta_{u} . \theta$ is obviously continuous on $M_{q}-K_{q}$. 
Furthermore, denote by $p_{u v}$ the projection sending $(u, v, w)$ into $(u, v, 0)$ and observe that $p_{u}=p_{u} p_{u v} \theta$ on $M_{q}-K_{q}$. Therefore, $\phi=p_{u} \psi$ $=p_{u}\left(p_{u v} \theta \psi\right)$ on $C$. Since $p_{u v} \theta \psi: C \rightarrow K_{q}$ is continuous, we have arrived at a contradiction with Lemma 6 . In a similar fashion we prove that $p_{w} \psi(t) \neq 1$, for all $t \in C$, is not possible.

4. Proof of Lemma 4. Let $C$ be an ordered continuum and let $\phi: C \rightarrow I$ be a map with $\phi\left(c_{0}\right)=0, \phi\left(c_{1}\right)=1$. Let

$$
t_{n}^{\prime}=\max \phi^{-1}\left(p_{u}\left(a_{n}\right)\right) \text {. }
$$

Then $\phi\left(\left[t_{n}^{\prime}, c_{1}\right]_{C}\right)=\left[p_{u}\left(a_{n}\right), 1\right]_{I}$. Set

$$
t_{n}^{\prime \prime}=\min \left(\phi^{-1}\left(p_{u}\left(a_{n+1}\right)\right) \cap\left[t_{n}^{\prime}, c_{1}\right]_{c}\right) .
$$

Since $p_{u}\left(a_{n+1}\right) \in\left[p_{u}\left(a_{n}\right), 1\right]_{I}, t_{n}^{\prime \prime}$ exists and we have

$$
\begin{aligned}
& t_{n}^{\prime}<t_{n+1}^{\prime}, \\
& t_{n}^{\prime}<t_{n}^{\prime \prime} \leqq t_{n+1}^{\prime} .
\end{aligned}
$$

Let $C_{n}=\left[t_{n}^{\prime}, t_{n}^{\prime \prime}\right]_{C}$ and let $\phi_{n}=\phi \mid C_{n} . C_{n}, n=0,1, \cdots$, are ordered continua and $\phi_{n}: C_{n} \rightarrow I_{n}=\left[p_{u}\left(a_{n}\right), p_{u}\left(a_{n+1}\right)\right]_{I}$ satisfies $\phi_{n}^{-1}\left(p_{u}\left(a_{n}\right)\right)=t_{n}^{\prime}$ and $\phi_{n}^{-1}\left(p_{u}\left(a_{n+1}\right)\right)=t_{n}^{\prime \prime}$. Denote by $\mu_{n}$ the mapping sending $u \in I_{n}$ into $\left(u, p_{v}\left(a_{n}\right), 0\right) \in I^{3}$ and consider $\lambda_{n}^{-1} \mu_{n} \phi_{n}: C_{n} \rightarrow I=I \times 0 \times 0$. For each $n \in\{0,1, \cdots\}$ choose such a $q(n)$ that

$$
0<2 q(n)<1-\eta\left(\lambda_{n}^{-1} \mu_{n} \phi_{n}\right) \leqq 1 .
$$

The claim is that there is no continuous mapping $\psi: C \rightarrow N_{(q(n))}$ with $p_{u} \psi=\phi$, i.e., that $r^{\prime}=(q(n)) \in R$ satisfies the requirements of Property (i).

Assume on the contrary that such a $\psi$ exists and denote $\psi \mid C_{n}$ with $\psi_{n}$. Then $\psi_{n}\left(C_{n}\right) \subset N_{(q(n))} \cap p_{u}^{-1}\left(I_{n}\right)=\lambda_{n}\left(M_{q(n)}\right)$ and $p_{u} \psi_{n}=\phi_{n}$. This implies

$$
p_{u}\left(\lambda_{n}^{-1} \psi_{n}\right)=\lambda_{n}^{-1} \mu_{n} p_{u} \psi_{n}=\lambda_{n}^{-1} \mu_{n} \phi_{n}
$$

and

$$
\left(\lambda_{n}^{-1} \psi_{n}\right)\left(C_{n}\right) \subset M_{q(n)} .
$$

Applying Lemma 7 to $\lambda_{n}^{-1} \mu_{n} \phi_{n}$, we conclude that there exist two points $\tau_{n}^{\prime}$ and $\tau_{n}^{\prime \prime}$ such that

$$
\begin{aligned}
\left\{\tau_{n}^{\prime}, \tau_{n}^{\prime \prime}\right\} \subset C_{n} & =\left[t_{n}^{\prime}, t_{n}^{\prime \prime}\right]_{c}, \\
\left(p_{w} \lambda_{n}^{-1} \psi_{n}\right)\left(\tau_{n}^{\prime}\right) & =\left(p_{w} \psi_{n}\right)\left(\tau_{n}^{\prime}\right)=0,
\end{aligned}
$$

and

$$
\left(p_{w} \psi_{n}\right)\left(\tau_{n}^{\prime \prime}\right)=1
$$


By (9), (10) and (12), there is a common limit $\tau$ for the two sequences $\tau_{n}^{\prime}$ and $\tau_{n}^{\prime \prime}$. However, (13) and (14) prevent $p_{w} \psi$ from being continuous, which contradicts the assumption that $\psi$ is continuous. This establishes Lemma 4. All that remains to do in order to complete the proof of Theorem 1 is to prove

Lemma 8. The family $\left\{S_{r}, \rho_{r}\right\}, r \in R$, defined above possesses Property (ii).

We know already that all $\rho_{r}=p_{u} \mid N_{(q(n))}$ are monotone and that all $S_{r}$ are continuous curves. We also know that $S_{r(1)} \ldots r(n)$ are metric compacta and admit monotone mappings onto $I$ (see $\$ 2,3$ ), which implies the connectedness of $S_{r(1)} \ldots r(n)$. Thus, it remains to show the local connectedness of $S_{r(1)} \ldots r(n)$.

4. Local connectedness of $S_{r(1) \cdots r(k)}$.

1. Let $\{r(1), \cdots, r(k)\}$ be a finite subset of $R$, where $r(i)$ is a sequence $(q(i, n)), n=0,1, \cdots$ In order to simplify notations, from now on we denote $r(i)$ simply by $i$ and $S_{r(1)} \ldots r(k)$ by $S_{1} \ldots k$. Let $s=s_{1} \times \cdots \times s_{k} \in S_{1} \ldots k \subset S_{1} \times \cdots \times S_{k}$ be arbitrarily chosen. We wish to find arbitrarily small connected neighborhoods of $s$ having the form $\left(G_{1} \times \cdots \times G_{k}\right) \cap S_{1 \ldots k}$, where $G_{i} \subset I^{3}$ are open sets and $s_{i} \in G_{i}$.

Consider a rectangle $P_{i}=U_{i} \times V_{i} \times p_{w}\left(s_{i}\right) \subset I^{3}$, where $U_{i}$ and $V_{i}$ are open connected sets of $I$ and $s_{i} \in P_{i}$. Let $G_{i}$ be an (open) parallelepiped from $I^{3}$, obtained as follows. First, translate $P_{i}$ in the direction of the $w$ axis (in positive and negative sense) to obtain an infinite prism $P^{\prime}$. In order to obtain a second prism $P^{\prime \prime}$, translate $P_{i}$ in the direction of a straight line lying in the plane $u=0$ and having a slope equal to 2 (slope with respect to the axes $v$ and $w$ ). Then set $G_{i}=P^{\prime} \cap P^{\prime \prime} ; P_{i}$ is a diagonal section of $G_{i}$.

Observe that $G_{i} \cap S_{i}=G_{i} \cap N_{(q(i, n))}$ is the union of a family of straight line segments (open or possibly half open) lying in the planes $u=$ constant and intersecting $P_{i}$. Consequently, each point $s_{i}^{\prime}$ $\in G_{i} \cap S_{i}$ can be joined, by a straight line segment lying in the plane $u=p_{u}\left(s_{i}^{\prime}\right)$, to a point $s_{i}^{\prime \prime} \in P_{i}$. This proves that each $s^{\prime} \in\left(G_{1} \times \cdots \times G_{k}\right) \cap S_{1} \ldots k$ can be joined, within the set $\left(G_{1} \times \cdots \times G_{k}\right) \cap S_{1 \ldots k}$, to a point $s^{\prime \prime} \in\left(P_{1} \times \cdots \times P_{k}\right) \cap S_{1} \ldots k$. Notice that $\left(P_{1} \times \cdots \times P_{k}\right) \cap S_{1} \ldots k$ is precisely the space $S\left(P_{i} \cap S_{i}, p_{u}\right)$, where $p_{u}$ stands for all the maps $p_{u}: P_{i} \cap S_{i} \rightarrow I$.

All this means that, in order to establish local connectedness of $S_{1} \ldots k$, it suffices to show that, for any given $s=s_{1} \times \cdots \times s_{k} \in S_{1 \ldots k}$, one can choose arbitrarily small rectangles $P_{1}, \cdots, P_{k}$ so that $S\left(P_{i} \cap S_{i}, p_{u}\right)$ be connected, $i=1, \cdots k$. 
2. The above stated task is an easy one if $p_{u}\left(s_{1}\right)=\cdots=p_{u}\left(s_{k}\right)<1$ since an inspection of the sets $P_{i} \cap S_{i}$ shows that, for sufficiently small $P_{i}, P_{i} \cap S_{i}$ is either a line segment or a very simple graph consisting of 2 or 3 edges meeting in one vertex. In order to settle the case $p_{u}\left(s_{1}\right)=\cdots=p_{u}\left(s_{k}\right)=1$, consider the rectangles $P_{i}$ for which the points $a_{n} \times p_{w}\left(s_{i}\right)$ and $\left(1,1, p_{w}\left(s_{i}\right)\right)$ form a pair of opposite vertices. Choose the same $n$ for all $i=1, \cdots, k$.

Clearly, the set $S\left(P_{i} \cap S_{i}, p_{u}\right)$ is the union of the sets $S_{m}$ $=S\left(\left(Q_{m} \times p_{w}\left(s_{i}\right)\right) \cap S_{i}, p_{u}\right), m \geqq n$, and of a single limit point $\left(a_{\infty} \times p_{w}\left(s_{1}\right)\right) \times \cdots \times\left(a_{\infty} \times p_{w}\left(s_{k}\right)\right)$. Since $S_{m}$ and $S_{m+1}$ have one point in common, it suffices to show that all $S_{m}$ are connected.

Notice that the sets $\left(Q_{n} \times p_{w}\left(s_{i}\right)\right) \cap S_{i}$ look just like the broken line $K_{q} \subset I^{2} \times 0$ (see $\S 3$ ), the only difference being that there are two additional straight line segments joining $b$ with a point $e=\left(q, q^{\prime}, 0\right)$ and $c$ with a point $f=\left(1-q, 1-q^{\prime}, 0\right), 0<q^{\prime}<1$. We denote the union of these segments and $K_{q}$ by $H_{q q^{\prime}}$ or merely by $H_{q}$. Thus, we have reduced our task to showing that the space $\Sigma=S\left(H_{q(i)}, p_{u}\right), i=1, \cdots, k$, is connected.

3 . The proof that $\Sigma$ is connected is by induction on $k$. Assume the assertion true for less than $k$ sets $H_{q(i)}$. Also assume that $q(1) \leqq \ldots$ $\leqq q(k)$. Let $\Sigma^{\prime}=S\left(H_{q(i)}, p_{u}\right), i=1, \cdots, k-1$. Let $\zeta: \Sigma \rightarrow I$ and $\zeta^{\prime}: \Sigma^{\prime} \rightarrow I$ be the maps induced by $p_{u}$ (see $\$ 2.1$ ). Then $\Sigma=S\left(\Sigma^{\prime}, H_{q(k)}, \zeta^{\prime}, p_{u}\right)$.

Denote by $\sigma_{0}^{\prime}$ the point $(q(k), 0,0) \times \cdots \times(q(k), 0,0) \in \Sigma^{\prime}$ $\subset H_{q(1)} \times \cdots \times H_{q(k-1)}$ and by $\sigma_{1}^{\prime}$ the point $(1-q(k), 0,0) \times \cdots$ $\times(1-q(k), 0,0) \in \Sigma^{\prime}$. By assumption, each point $\sigma^{\prime} \in \Sigma^{\prime}$ $\cap \zeta^{\prime-1}\left([q(k), 1-q(k)]_{I}\right)$ can be joined, within that set, to the point $\sigma_{0}^{\prime}$ as well as to $\sigma_{1}^{\prime}$.

Let $s_{k} \in H_{q(k)}$ be such that $\sigma^{\prime} \times s_{k} \in \Sigma \subset \Sigma^{\prime} \times H_{q(k)}$. Then $s_{k}$ lies on one of these five segments: $b g, b e, b c, c f, c h$, where $g=\left(p_{u}(c), 0,0\right), h$ $=\left(p_{u}(b), 1,0\right)$. It is clear that, given any path connecting $\sigma^{\prime}$ and $\sigma_{0}^{\prime}$ in $\Sigma^{\prime} \cap \zeta^{\prime-1}\left([q(k), 1-q(k)]_{I}\right)$, one can vary the point $s_{k}$ along one of these five segments in such a fashion that we have all the time $\zeta^{\prime}\left(\sigma^{\prime}\right)=p_{u}\left(s_{k}\right)$. In other words, $\sigma^{\prime} \times s_{k}$ can be connected, within $\Sigma$, to one of these three points: $\sigma_{0}^{\prime} \times g, \sigma_{0}^{\prime} \times e, \sigma_{0}^{\prime} \times c$.

Similarly, varying $\sigma^{\prime}$ in $\Sigma^{\prime}$ and $s_{k}$ along $c b$, one can join $\sigma_{0}^{\prime} \times c$ and $\sigma_{1}^{\prime} \times b$ within $\Sigma$. Furthermore, $\sigma_{1}^{\prime} \times b$ can be joined with $\sigma_{0}^{\prime} \times g$. A similar argument applies to $\sigma_{0}^{\prime} \times e$ and $\sigma_{0}^{\prime} \times g$. The conclusion is that all points of $\Sigma \cap\left(\zeta^{-1}\left([q(k), 1-q(k)]_{I}\right)\right)$ belong to the same component of $\Sigma$ as $\sigma_{0}^{\prime} \times g$ and $\sigma_{1}^{\prime} \times h$. On the other hand, it is immediate that the remaining points of $\Sigma$ are easily connected (within $\Sigma$ ) with $\sigma_{0}^{\prime} \times g$ or $\sigma_{1}^{\prime} \times h$. This concludes the proof that $\Sigma$ is connected. 
REMARK. If $X_{1}, X_{2}$ are two continuous curves and $\rho_{i}: X_{i} \rightarrow I, i=1,2$, are monotone mappings onto, then $S\left(X_{1}, X_{2}, \rho_{1}, \rho_{2}\right)$ need not be locally connected.

\section{REFERENCES}

1. C. E. Capel, Inverse limit spaces, Duke Math. J. vol. 21 (1954) pp. 233-246.

2. H. Hahn, Mengentheoretische Charakterisierung der stetigen Kurve, Sitzungsber. Akad. Wiss., Wien, vol. 123 (1914) pp. 2433-2489.

3. J. L. Kelley, General topology, New York, van Nostrand Co., 1955. 209.

4. S. Mazurkiewicz, Sur les lignes de Jordan, Fund. Math. vol. 1 (1920) pp. 166-

5. R. L. Wilder, Topology of manifolds, Amer. Math. Soc. Colloquium Series, vol. 32, New York, 1949.

The University of Zagreb, Zagreb, Yugoslavia

\section{A CLAN WITH ZERO WITHOUT THE FIXED POINT PROPERTY}

\section{HASKELL COHEN}

There is a conjecture due to A. D. Wallace that a clan (i.e., a compact, connected, topological semigroup with identity element) with a zero element has the fixed point property. This is related to another conjecture of Wallace that a compact connected topological lattice has the fixed point property [4]. A proof of the latter conjecture for the finite dimensional case has recently been given by Dyer and Shields [1]. There is an example due to Kinoshita [2] of a contractable continuum without the fixed point property. The purpose of this note is to exhibit a multiplication which will make Kinoshita's example into a clan with zero, and, thus, provide a counter example to the first conjecture above.

We exhibit first a result which seems to be rather generally known, but which, to the author's knowledge, does not appear in print.

Lemma. Suppose $S$ is a topological semigroup, and $f$ is an open or closed map taking $S$ onto $T$, a Hausdorff space. Suppose further that $f(a)=f(b)$ and $f(c)=f(d)$ implies $f(a c)=f(b d)$. Then $T$ can be given $a$ multiplication which makes it a topological semigroup and which makes f a homomorphism.

Presented to the Society, November 19, 1960; received by the editors January 29, 1960. 